\title{
Comparison of exposure levels of carotid artery in T1-2 glottic cancer patients undergoing radiotherapy with different modalities: A simulation-based dosimetric study
}

\author{
Gonca Hanedan Uslu', Emine Canyilmaz ${ }^{2}$, Fatma Colak ${ }^{2}$, Burcin Hazeral ${ }^{3}$, Ahmet Yasar Zengin ${ }^{1}$, Hüseyin Göçmez ${ }^{1}$ and Adnan Yoney ${ }^{2}$ \\ ${ }^{1}$ Department of Radiation Oncology, Kanuni Research and Education Hospital, Trabzon, Turkey \\ ${ }^{2}$ Department of Radiation Oncology, Faculty of Medicine, Karadeniz Technical University, Trabzon, Turkey \\ ${ }^{3}$ Onkomer Oncology Center, İzmir,Turkey
}

\begin{abstract}
Limiting the unwanted exposure level of neighboring healthy tissues including carotid artery is important with respect to carotid artery atherosclerosis risk, which could lead to cerebrovascular events, in patients undergoing radiotherapy for neck and head malignancies. The aim of this study was to compare carotid artery irradiation exposure levels in T1-2 glottic cancer patients undergoing radiotherapy with different modalities namely three dimensional conformal radiotherapy (3DCRT-opposed laterals field $(O L F)$, three field $(3 F)$, intensity-modulated radiotherapy (IMRT) and intensity-modulated arc therapy (IMAT). The radiation exposure level of carotid artery was determined by use of InDigital Imaging and Communications in Medicine radiotherapy (DICOM-RT) datasets. This simulation-based dosimetric analysis was performed by use of DICOM-RT data for seven T1-2 glottic squamous cell carcinoma patients who received conventional 66Gy dose in 33 fractions, and levels of carotid exposure for 3DCRT, IMRT and IMAT techniques was calculated for each patient. The calculations involved PTV D95, PTV Dmax, left and right carotid artery doses $\mathrm{V} 35, \mathrm{~V} 50$ and $\mathrm{V} 63 \mathrm{~Gy}$, conformity index (CI), homogeneity index (HI) and monitor unit (MU) for each plan. Effective target volume was ensured with all the techniques tested. IMAT plan yielded the lowest carotid artery exposure as values of V35, V50 and V63 for carotid artery were lowest in IMAT plans. PTV V95, HI values of IMRT plans were significantly better. CI values for 3DCRT-3F, IMRT and IMAT plans were not significantly different, all being significantly lower than 3DCRT-OFL. The results of this pilot study indicates that IMAT plan provides the lowest risk of carotid artery exposure and thereby related complications in treatment of T1-2 glottic cancers with radiotherapy.
\end{abstract}

\section{Introduction}

Risk of having stroke is higher in the patients that received local RT for head and neck cancers [1]. However, the mechanisms underlying this condition are not clear; atherosclerosis of the veins in the RT region plays a crucial role. This mechanism plays an important role especially in addiction of nicotine.

Early stage T1-T2N0M0 glottic cancers are highly curable when they are treated with RT $[2,3]$. Because of lower nodal involvement rates elective nodal irradiation is not necessary. Therefore acute and late toxicities are lower [4]. RT related vascular toxicities usually observed after 10 years. Prevalence of stroke and cerebrovascular accident because of RT to the neck region is between $2.5-12 \%[5,6]$. It's known that conventional irradiation with parallel-opposed fields especially increases carotid artery doses [7]. Increased doses causes intima-media thickening in arteries and this leads to arteriosclerosis. Results of Color Doppler tests before RT and one year after RT have shown that there is a significant luminal narrowing in veins [8].

In today's world conformal radiotherapy is being used rather than parallel-opposed fields but intensity modulated radiotherapy (IMRT) is widely used treatment modality among all. By using treatment plans that used IMRT technique sharp dose drop can be achieved between glottic clinical target volume and carotid artery. More recently, three reports similarly observed a better target coverage and significant dose reduction to the carotid arteries compared to conventional techniques, suggesting that IMRT may decrease the rate of carotid atherogenesis in the future $[3,9,10]$.

Identification of RT technique that gives the lowest carotid artery doses important in decreasingthe risk of RT caused strokes and cerebrovascular events especially in patients with nicotine addiction.

In the present study, we compared the dosimetric results of IMAT, which delivers highly conformal IMRT plans in a short time, to threedimensional conformal radiotherapy (3DCRT-OLF, 3F) and classic IMRT techniques. We compared dosimetric features of four techniques in terms of target coverage, exposure to the carotid artery, conformity index (CI), homogeneity index (HI).

\section{Patients and methods}

In our study, we used the computed tomography (CT) images of 7 early staged larynx cancer (T1-2 N0) patients who had irradiation

Correspondence to: Gonca Hanedan Uslu, MD, Department of Radiation Oncology, Kanuni Research and Training Hospital, Topal Osman Street, No: 7, Kasüstü - Yomra/Trabzon 60080, Turkey, Tel: +90 046223023 00, Fax: +90 0462 23023 07; E-mail: drgoncahanedanuslu@hotmail.com

Key words: carotid artery, glottic cancer, IMRT, IMAT, radiotherapy techniques Received: Janaury 10, 2015; Accepted: January 28, 2016; Published: January 30, 2016 
with conventional 3DCRT. $2.5 \mathrm{~cm}$ sliced thickness actual treatment images which was acquired with GE Optima CT-Simulator has been transferred into Eclipse treatment system. All target volumes and organs at risk (OAR) volumes have been defined.

In order to use these data in this study ethic board permission from the same university has been taken.

\section{Volume definitions}

Clinical target volume (CTV) was contoured by the same physician to encompass the thyroid with a 5-mm margin anteriorly, cricoids cartilages, arytenoid cartilage, false vocal cords, anterior and posterior commissures, true vocal cords and $1-1.5 \mathrm{~cm}$ of subglottis; the borders were extended to the hyoid bone superiorly and to the bottom of the cricoids inferiorly. Each CTV was modified to encompass predelineated gross tumor volume. CTV was truncated within $3 \mathrm{~mm}$ of the skin surface to avoid high skin dose in patients without anterior commissure involvement. Planning target volumes (PTV) was created with giving $5 \mathrm{~mm}$ margin around the CTV. The spinal cord and carotid arteries were defined as the critical structures. Organ at risk (OAR) volumes of spinal cord and carotid arteries were delineated to exceed PTV by $1 \mathrm{~cm}$ on superior and inferior directions.

\section{Treatment planning and prescription dose}

For each 7 patients 4 different plan (3DCRT-OLF, 3F, IMRT and IMAT) and in total 28 new planning have been done. In all treatment techniques $66 \mathrm{~Gy}$ (2Gy/fraction) treatment modality has been used. Six MV photon beams and a dynamic multi-leaf collimator (DHX equipped with a Millenium-120 MLC, spatial resolution of $5 \mathrm{~mm}$ at the isocenter) were used for each plan. The doses for the CTV, left and right carotid arteries and spinal cord and monitor units (MU) were calculated for each plan.

Dose volume histograms (DVH) were used in dose comparison. Dmean (mean dose), Dmax (maximum dose), V95: 62.7Gy, conformity index (CI) and homogeneity index (HI) values which belong to PTV have been used in planning comparisons. Threshold dose for intimamedia layer of carotid artery was determined as $>35-50 \mathrm{~Gy}$ [11]. For that reason in planning comparisons $35 \mathrm{~Gy}, 50 \mathrm{~Gy}$ and 63Gy reference range and V35 (percentage of volume receiving $35 \mathrm{~Gy}$ ), V50 (percentage of volume receiving $50 \mathrm{~Gy}$ ) andV63 (percentage of volume receiving 63 Gy), Dmean, Dmax values has been used. All plans were normalized so that $>95 \%$ of the CTV received $100 \%$ of the prescription dose.

3DCRT planning: 2 plans that uses Opposed laterals field $(O L F)$ and three field plan $(3 F)$ have been done and for OLF $90^{\circ}$ and $270^{\circ}$ gantry angles have been used. In order to obtain dose homogeneity in treatment fields wedges have been used with collimation. Also in $3 \mathrm{~F}$ planning $0^{\circ}, 90^{\circ}$ and $270^{\circ}$ gantry angles have been used and in order to obtain dose homogeneity wedges have been used. Full dose (66Gy) has been given to $95 \%$ of the PTV volume.

IMRT planning: 5 field IMRT plan has been used in all 5 patients. The IMRT plan set used five non-equally spaced beams, which were isocentrically centered on the CTV $\left(0^{\circ}, 51^{\circ}, 102^{\circ}, 255^{\circ}\right.$ and $\left.306^{\circ}\right)$. In optimization process a new PTV has been defined by giving $1 \mathrm{~mm}$ safety margin to PTV. Full dose (66Gy) has been given to $95 \%$ of the PTV volume. Tried to be unsure that critical organs were within tolerance doses. All beams were collimated to minimize exposure to the carotid arteries. The dose homogeneity goal was $<110 \%$ within the CTV

IMAT planning: In IMAT plans $\mathrm{P} 35^{\circ}$ collimation between 182 178, 178-182, 263-95 gantry angles with 3 field arcs has been used. 6
MV photon energy has been used. IMAT treatment fields two $356^{\circ}$ arcs have been divided into 177 control points and $168^{\circ}$ arc have been divided into 129 control points. For each control point beam range has been defined with changes in multi leaf collimator and gantry angles. Dose rate was between 0 and $600 \mathrm{MU} / \mathrm{min}$ and gantry rotation was between $0,0^{\circ} / \mathrm{S}$ and $4,8^{\circ} / \mathrm{S}$. In order to minimize tongue and groove effect, $35^{\circ}$ rotation to the collimator was given during treatment. After many plans that uses one, double and 2.5 arcs best plan to use was 2.5 arc IMAT. In order to protect normal tissues a new volume $3 \mathrm{~mm}$ outside of PTV was defined. Also a new volume has been created for critical organs, which were in PTV volume. During optimization process more protection has been ensured for the field $3 \mathrm{~mm}$ outside of PTV. By this way maximum protection has been done for critical organs in the field. After first optimization process undesirable dose areas, exceeds in maximum doses and cold spots in PTV have been defined as new volumes. These volumes have been used in the second optimization process in order to obtain homogenous dose distribution and better critical organ dose rates.

\section{Plan evaluation}

Plans were compared in terms of PTV coverage (D95=dose defined to $95 \%$ of the PTV), maximum point dose within the PTV (Dmax=maximum point dose in one point), total MU, CI, HI and carotid artery dose.

\section{The CI of the PTV was defined as:}

$$
\mathrm{CI}=\mathrm{V}_{\mathrm{PTV}} \times \mathrm{V}_{\mathrm{TV}} / \mathrm{TV}_{\mathrm{PV}}^{2}
$$

VPTV: Volume of PTV, VTV: treatment volume of prescribed isodose lines, TVPV2: volume of PTV within VTV

The HI of the PTV was defined as:

\section{$\mathrm{HI}=\mathrm{D} 5 \% / \mathrm{D} 95 \%$}

D5\%; minimum dose in 5\% of the Planning Target Volume (PTV), D95\%; minimum dose in $95 \%$ of the PTV

\section{Statistical analysis}

ANOVA, post hoc Tukey test for parametrically distributed data was used to compare the results between IMRT, VMAT and 3DCRT. All statistical tests were two-sided, with a threshold for statistical significance of $\mathrm{P}<0.05$. Statistical analysis was carried out using SPSS version 13.

\section{Results}

Dose-volume data for the PTV's are given in Table 1. The calculated D95 values for each of the 4 techniques ranged between $63.95 \mathrm{~Gy}$ and 66.2 Gy. Best value has been obtained from IMPRT plan and this was statistically significant $(p=0.001)$. Dmax for the 10 patients and 4 plans, ranged between $70.90 \mathrm{~Gy}$ and $71.70 \mathrm{~Gy}$ for all plans. No difference was detected between 4 techniques $(p=0.08)$. Dmax values for IMRT and IMAT techniques were significantly higher than 3D-CRT plans $(p=0.008)$.

Dose-volume parameters for left and right carotid arteries for each treatment techniques are given in table 2. Carotid arteries mean dose, V35 and V50 were significantly high for 3DCRT-OLF and 3DCRT-3F plans compared to IMRT and IMAT plans. Lowest dose values obtained from IMAT plans and this was statistically significant $(p=0.001)$. Spinal cord maximum point median dose was $4.6 \pm 2.5$ Gy (range: $2.29-10.21 \mathrm{~Gy}$ ) for 3DCRT-OLF plans. For IMRT 
Uslu GH (2016) Comparison of exposure levels of carotid artery in T1-2 glottic cancer patients undergoing radiotherapy with different modalities: A simulationbased dosimetric study

Table 1. Dosimetric parameters for target volume for all plans.

\begin{tabular}{|c|c|c|c|c|c|}
\hline & 3DCRT-OFL & 3DCRT-3F & IMRT & IMAT & $\mathbf{p}$ \\
\hline PTV66 Mean Dose (Gy) & $68.13 \pm 0.2$ & $68.07 \pm 0.4$ & $68.65 \pm 0.4$ & $67.05 \pm 0.3$ & 0.001 \\
\hline PTV66 Max Dose (Gy) & $70.90 \pm 0.3$ & $71.11 \pm 0.6$ & $71.70 \pm 0.5$ & $71.33 \pm 0.6$ & 0.08 \\
\hline D\%95 (Gy) & $65.03 \pm 0.5$ & $64.64 \pm 0.2$ & $66.02 \pm 0.4$ & $63.95 \pm 0.9$ & 0.001 \\
\hline D\%5 (Gy) & $70.25 \pm 0.3$ & $70.50 \pm 0.6$ & $70.52 \pm 0.5$ & $69.02 \pm 0.3$ & 0.001 \\
\hline HI & $1.08 \pm 0.006$ & $1.08 \pm 0.01$ & $1.06 \pm 0.007$ & $1.07 \pm 0.01$ & 0.01 \\
\hline CI & $1.9 \pm 0.2$ & $1.4 \pm 0.05$ & $1.3 \pm 0.07$ & $1.3 \pm 0.1$ & 0.001 \\
\hline MU & $243 \pm 0.02$ & $242 \pm 0.08$ & $705 \pm 0.9$ & $915 \pm 0.9$ & 0.001 \\
\hline
\end{tabular}

PTV: Planinig Target Volüme, HI: Homojenite İndex , CI: Conformalite İndex , MU: Monutor Unit

Table 2. Dosimetric parameters for bilateral carotid arteries and MS for all plans.

\begin{tabular}{|c|c|c|c|c|c|}
\hline & 3DCRT-OFL & 3DCRT-3F & IMRT & IMAT & $\mathbf{p}$ \\
\hline RCA Mean dose (Gy) & $55.39 \pm 4.2$ & $43.54 \pm 3.7$ & $27.63 \pm 5.9$ & $17.72 \pm 2.1$ & 0.001 \\
\hline LCA Mean dose (Gy) & $55.81 \pm 3.9$ & $47.30 \pm 5.1$ & $32.39 \pm 5.5$ & $22.92 \pm 5.4$ & 0.001 \\
\hline RCA Max dose (Gy) & $69.07 \pm 0.6$ & $63.88 \pm 2.5$ & $64.75 \pm 4.7$ & $59.29 \pm 6.9$ & 0.004 \\
\hline LCA Max dose (Gy) & $68.98 \pm 0.4$ & $65.73 \pm 2.4$ & $66.68 \pm 4.2$ & $65.00 \pm 5.06$ & 0.1 \\
\hline RCA V35 (\%) & $83.00 \pm 6.2$ & $75.52 \pm 3.7$ & $33.35 \pm 15.7$ & $7.1 \pm 5.5$ & 0.001 \\
\hline LCA V35 (\%) & $83.22 \pm 6.0$ & $79.32 \pm 5.3$ & $45.38 \pm 13.9$ & $20.29 \pm 12.7$ & 0.001 \\
\hline RCA V50 (\%) & $75.24 \pm 6.8$ & $41.30 \pm 12.6$ & $13.28 \pm 11.2$ & $1.9 \pm 3.09$ & 0.001 \\
\hline LCA V50 (\%) & $75.87 \pm 0.5$ & $58.38 \pm 14.1$ & $21.82 \pm 11.2$ & $8.8 \pm 11.1$ & 0.001 \\
\hline RCA V63 (\%) & $59.01 \pm 9.1$ & $2.6 \pm 5.6$ & $1.6 \pm 2.1$ & $0.4 \pm 1.14$ & 0.001 \\
\hline LCA V63 (\%) & $62.22 \pm 6.7$ & $13.3 \pm 16.2$ & $4.5 \pm 5.3$ & $3.2 \pm 6.5$ & 0.001 \\
\hline MS Max & $4.6 \pm 2.5$ & $21.4 \pm 0.9$ & $41.4 \pm 5.9$ & $44.24 \pm 1.8$ & 0.001 \\
\hline
\end{tabular}

RCA: Sağ Karotis Arter, LCA: Sol Karotis Arter, MS: Medullaspinalis

and IMAT plans, respective spinal cord maximum doses were 41.4 \pm 5.9 and $44.24 \pm 1.8$, which were significantly higher than 3DCRTOLF and 3DCRT- $3 F$ plans (Table 2). However, all values were below the critical clinical threshold dose of $45 \mathrm{~Gy}$.

The calculated MU per fraction values for each technique are given in Table 1. Among all plans, the highest MU was obtained for the IMAT plan. Mean MU values for IMRT and IMAT plans were $705 \pm$ 0.9 and $915 \pm 0.9 \mathrm{MU} / \mathrm{min}$, respectively. Mean MU values for 3DCRTOFL and 3DRT-3F plans were $243 \pm 0.02$ and $242 \pm 0.08 \mathrm{MU} / \mathrm{min}$, respectively. The MU was significantly lower with than IMRT with IMAT. Highest MU has been observed in IMAT plan and lowest MU has been obtained from 3DCRT-OLF ( $p=0.001)$.

Best HI values were obtained from IMRT but this was statistically significant (Table 1). The HI values ranged between 1.08 and 1.06 and this was significantly different between the 3 plans $(\mathrm{p}=0.01)$. There was no difference between IMRT and IMAT in terms of CI. Highest CI has been obtained from 3DCRT-OLFas 1.9 and lowest from IMAT as 1.3. These values were significantly different between the plans of $3 D C R T$ and IMRT and IMAT (Table 1, $p=0.001$ ).

\section{Discussion}

Radiotherapy is one of the treatment modality among many treatment options in early staged glottic laryngeal cancer. RT is the most efficient treatment option in terms of larynx protection. For many decades parallel opposed fields have been used for treatment but in recent years IMRT technique has been started to use in order to minimize permanent side effects like xerostomia, better normal tissue protection and maximum coverage for target volumes [2,12]. In our study comparing 3DCRT with IMAT and IMRT techniques, latter ones reveals better PTV coverage. A recent editorial called attention to a high number of patients requiring salvage laryngectomy after IMRT failure in early stage glottic cancer. The authors concluded that IMRT has little potential to provide significant benefits for patients and warned that its use may decrease local control rates [12].

Carotid artery doses were lower with IMRT [3]. Many studies has mentioned hazardous dose response value instead of carotid artery threshold dose. Martin et al. [11] suggested that thickness intimamedia was statistically meaningful in $\geq 35-50$ Gy. For this reason chosen reference dose-volume parameters for both carotids fractional volume identified as V 35 and V50. In our study best V 35 and V50 values obtained from IMAT compared to 3DCRT and IMRT. These values in our study were higher than many studies. Identifying PTV volumes as CTV $+0.5 \mathrm{~mm}$ in PTV dose calculations might be the reason for this. Rosenthal et al. [3] showed that it is possible to create sharp dose gradients between target volumes and carotid arteries with simulated IMRT plans. Also, they suggested that lowering carotid artery doses are necessary especially for young patients with carotid artery pathology. Chera et al. [9] compared radiation doses to carotid arteries among various radiotherapy techniques for treatment of early stage carcinoma of the glottis. They concluded that IMRT can reduce the dose to carotid arteries. However, they warned that the potential advantage of reducing carotid dose outweighs the risk of tumor recurrence due to contouring errors, organ motion and the risk of complications from dose heterogeneity.

There are few studies in the literature that investigates the place of IMAT technique in carotid artery protection [13,14]. A shorter treatment time may reduce the risk of target under-dosage due to organ motion might be the reason of this. Atalar et al. [15] indicated that shortest treatment duration has been obtained from IMAT technique and lowest carotid artery dose rated has been obtained from IMAT technique. In our study we also observed that carotid artery doses were lowest in IMAT plans compared with 3DCRT and IMRT. IMAT was given the best V35, V50, V63 values for right carotid artery were respectively 7.1Gy, 1.9 Gy, 0.4 Gy. Even though short treatment duration with IMAT compromised with target volume movement, this can be avoided by short duration swallow command. 
Uslu GH (2016) Comparison of exposure levels of carotid artery in T1-2 glottic cancer patients undergoing radiotherapy with different modalities: A simulationbased dosimetric study

\section{Conclusions}

In head and neck cancers radiotherapy constitutes a risk factor in terms of carotid artery atherosclerosis and cerebrovascular event. In this study comparing IMRT, 3DCRT and IMAT plans in terms of carotid artery doses has revealed that significant decrease in carotid artery doses acquired with IMAT technique. However, the potential increased rate of laryngeal complications from dose inhomogeneity should also be prospectively investigated.

\section{Contribution details}

GHU carried out the study concept, design, literature search and manuscript writing.

EC carried out the study design, literature search and manuscript preparation.

FC carried out the IMRT plans and data acquisition manuscript prepation.

$\mathrm{BH}$ carried out the IMAT plans and data acquisition manuscript prepation.

AYZ carried out the literature search.

HG carried out the literature search and manuscript writing.

AY carried out the manuscript editing and manuscript review.

\section{Acknowledgements}

We wish to thank Dr. Serdar Ozkok for their assistance in preparing this manuscript.

\section{References}

1. Dorresteijn LD, Kappelle AC, Scholz NM, Munneke M, Scholma JT, et al. (2005) Increased carotid wall thickening after radiotherapy on the neck. Eur J Cancer 41: 1026-1030.[Crossref]

2. Mlynarek A, Kost K, Gesser R (2006) Radiotherapy versus surgery for early T1-T2 glottic carcinoma. J Otolaryngol 35: 413-419.[Crossref]

3. Rosenthal DI, Fuller CD, Barker JL Jr, Mason B, Garcia JA, et al. (2010) Simple carotid-sparing intensity-modulated radiotherapy technique and preliminary experience for T1-2 glottic cancer. Int JRadiat Oncol Biol Phys 77: 455-461. [Crossref]

4. Mendenhall WM, Amdur RJ, Morris CG, Hinerman RW (2001) T1-T2N0 squamous cell carcinoma of the glottic larynx treated with radiation therapy. J Clin Oncol 19: 4029-4036.[Crossref]

5. Brown PD, Foote RL, McLaughlin MP, Halyard MY, Ballman KV, et al. (2005) A historical prospective cohort study of carotid artery stenosis after radiotherapy for head and neck malignancies. Int J Radiat Oncol Biol Phys63: 1361-1367.[Crossref]

6. Hong JC, Kruser TJ, Gondi V, Mohindra P, Cannon DM, et al. (2013) Risk of cerebrovascular events in elderly patients after radiation therapy versus surgery for early-stage glottic cancer. Int J Radiat Oncol Biol Phys 87: 290-296.[Crossref]

7. Faruolo M, Fiorentino A, Gallucci G, Lapadula L, Fusco V (2013) Intimal-media thickness and carotid arteries lumen in irradiated patients for head and neck cancer: preliminary data of an observational study. Clin Transl Oncol 15: 861-864.[Crossref]

8. Hardie CL, McKenna A, Przeslak AJ, Morgan DA (2007) Minimising carotid artery dose in the radiotherapy of early glottic cancer. ClinOncol (R CollRadiol) 19: 800 . [Crossref]

9. Chera BS, Amdur RJ, Morris CG, Mendenhall WM (2010) Carotid-sparing intensitymodulated radiotherapy for early-stage squamous cell carcinoma of the true vocal cord. Int J Radiat Oncol Biol Phys 77: 1380-1385.[Crossref]

10. Gomez D, Cahlon O, Mechalakos J, Lee N (2010) An investigation of intensitymodulated radiation therapy versus conventional two-dimensional and 3D-conformal radiation therapy for early stage larynx cancer. Radiat Oncol 26: 74. [Crossref]

11. Martin JD, Buckley AR, Graeb D, Walman B, Salvian A, et al. (2005) Carotid artery stenosis in asymptomatic patients who have received unilateral head and neck irradiation. Int J Radiat Oncol Biol Phys 63: 1197-205.[Crossref]

12. Feigenberg SJ, Lango M, Nicolaou N, Ridge JA (2007) Intensity-modulated radiotherapy for early larynx cancer: is there a role? Int J Radiat Oncol Biol Phys 68: 2-3.[Crossref]

13. Verbakel WF, Cuijpers JP, Hoffmans D, Bieker M, Slotman BJ, et al. (2009) Volumetric intensity- modulated arc therapy vs. conventional IMRT in head-and-neck cancer: A comparative planning and dosimetric study. Int J Radiat Oncol Biol Phys 74: 252-259. [Crossref]

14. Doornaert P, Verbakel WF, Bieker M, Slotman BJ, Senan S (2011) RapidArc planning and delivery in patients with locally advanced head-and-neck cancer undergoing chemoradiotherapy. Int J Radiat Oncol Biol Phys79: 429-435.[Crossref]

15. Atalar B, Gungor G, Caglar H, Aydin G, Yapici B, et al. (2012) Use of volumetric modulated arc radiotherapy in patients with early stage glottic cancer. Tumori 98 : 331 336.[Crossref]

Copyright: (C) 2016 Uslu GH. This is an open-access article distributed under the terms of the Creative Commons Attribution License, which permits unrestricted use, distribution, and reproduction in any medium, provided the original author and source are credited. 